\title{
Which Xenobiotic(s) Could be Responsible for the Radiologic Findings Below?
}

\author{
Answer: Any proconvulsant xenobiotic, in this case tramadol, bupropion, and nortriptyline
}

\section{Samuel J. Stellpflug • David J. Roberts}

Published online: 20 March 2010

(C) American College of Medical Toxicology 2010

\begin{abstract}
Background The proconvulsive properties of tramadol, bupropion, and nortriptyline have been well documented. Spinal fractures secondary to drug-induced seizures have been rarely reported.

Case Report A 39-year-old female presented with a chief complaint of back pain. She went to bed feeling well in a separate room from her husband. During the previous night, he heard a noise and went into her room, finding her confused and twisted in an awkward position on her bed. Later she complained to him of severe back pain, prompting transport to a hospital. Shortly after arrival in the emergency department, staff witnessed a generalized convulsion. Following a one-hour post-ictal period, she complained of worsened back pain. Lab studies were normal, including a urine tox screen for drugs of abuse. No alcohol was implicated. ECG showed sinus rhythm, HR 113 beats/min, QRS $108 \mathrm{~ms}$, QTc 389 ms. Brain magnetic resonance imaging (MRI) was normal. X-ray and an MRI of the thoracic spine confirmed four contiguous vertebral compression fractures, from T2 through T5. EEG showed diffuse changes consistent with a metabolic or toxicologic process. She denied taking any drugs other than prescribed doses of her medications, which included tramadol,
\end{abstract}

\section{J. Roberts}

North Memorial Medical Center Department of Emergency Medicine,

Robbinsdale, MN, USA

S. J. Stellpflug $(\bowtie) \cdot$ D. J. Roberts

Hennepin Regional Poison Center,

Minneapolis, MN, USA

e-mail: samuel.j.stellpflug@healthpartners.com

\section{S. J. Stellpflug}

Regions Hospital Department of Emergency Medicine and Clinical

Toxicology Service,

St. Paul, MN, USA bupropion, and nortriptyline. She had no previous history of seizures, head injury, or CVA. Bupropion and tramadol were discontinued, and seizures did not recur.

Case Discussion This patient's history, EEG findings, and brain imaging all point to a metabolic or toxic cause. It is likely that her three proconvulsant medications - even at therapeutic doses - synergistically lowered her seizure threshold or even precipitated her seizures. Retrospective studies and case reports portray these drugs as potentially offending agents.

Conclusions Sudden onset of back pain during sleep can be an important clue to a seizure complicated by vertebral

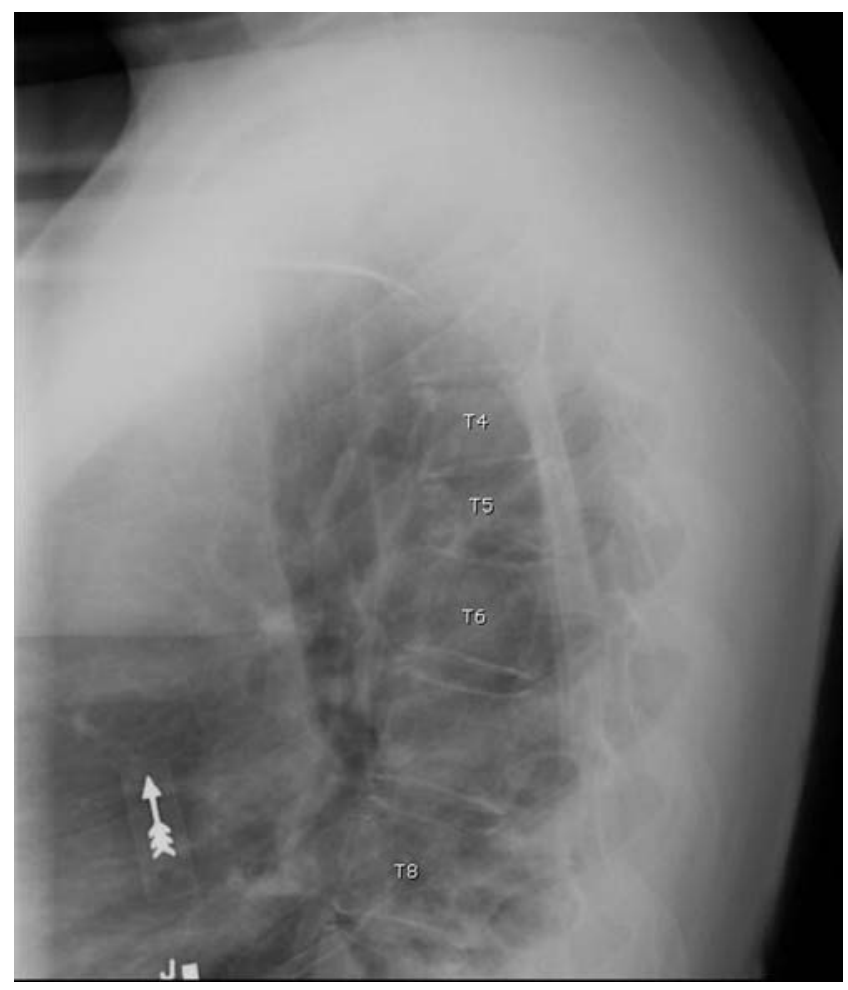

Fig. 1 X-ray, spine 


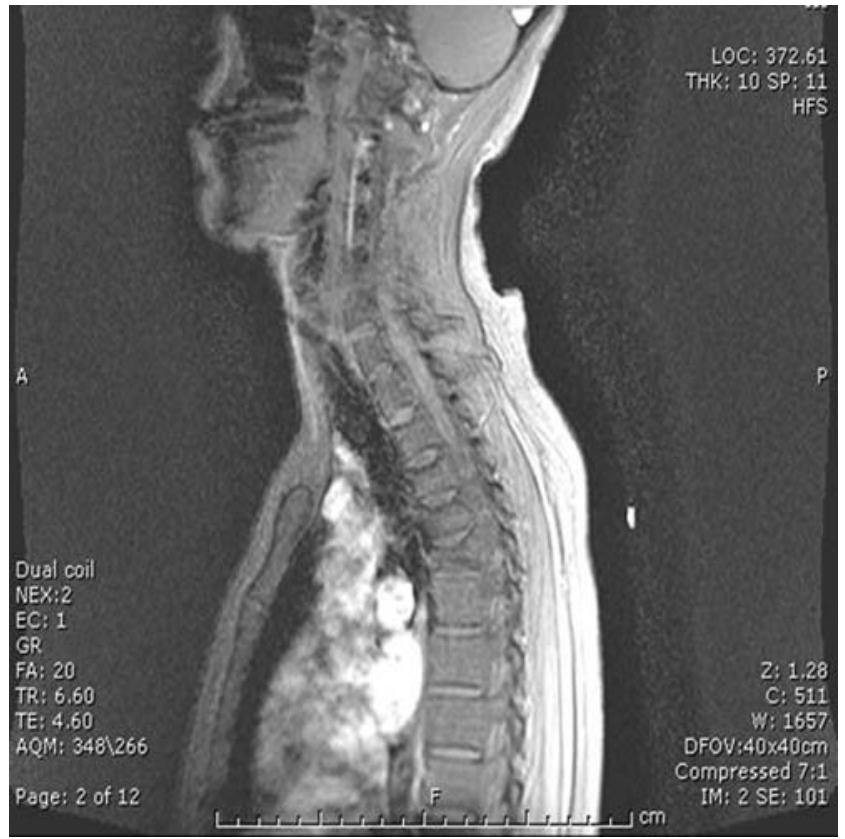

Fig. 2 Magnetic resonance imaging, spine

compression fractures, even in the absence of trauma. Toxicology consultation in seizures of unclear etiology can help discern drugs that offend even in therapeutic doses.

Keywords Proconvulsant · Bupropion .

Vertebral compression fracture

The proconvulsive properties of tramadol, bupropion, and nortriptyline have been well documented in overdose. Even in therapeutic dosing, they have been shown to lower the seizure threshold [1-3]. Spinal fractures secondary to druginduced seizures have been rarely reported $[4,5]$. We present a patient who suffered seizures-likely related to her medications - complicated by spinal fractures.

A 39-year-old female presented to the emergency department with a chief complaint of upper back pain. She had gone to bed feeling well in a separate room from her husband. During the night, he heard a noise and went into her room, finding her confused and twisted in an awkward position on her bed. Later she complained to him of severe back pain, prompting transport to a hospital. Shortly after arrival in the emergency department, staff witnessed a generalized convulsion. Following a 1-h post-ictal period, she complained of worsened back pain. Lab studies were essentially normal, including a urine tox screen for drugs of abuse. No alcohol was implicated. ECG showed sinus rhythm, heart rate of 113 beats/min, QRS $108 \mathrm{~ms}$, QTc $389 \mathrm{~ms}$. Brain magnetic resonance imaging (MRI) was normal. A toxicologist was consulted, and he offered assistance in the workup and management of the seizures and back pain. X-ray (Fig. 1) and MRI (Fig. 2) scans of the thoracic spine confirmed four contiguous vertebral compression fractures, from T2 through T5. EEG showed diffuse changes consistent with a metabolic or toxicologic process. She denied taking any drugs other than prescribed doses of her medications, which included tramadol, bupropion, and nortriptyline. She had no previous history of seizures, head injury, or CVA. Bupropion and tramadol were discontinued, and seizures did not recur in the short follow-up period available. This patient's history, EEG findings, and brain imaging all point to a metabolic or toxic cause. It is likely that her three proconvulsant medicationseven at therapeutic doses-synergistically lowered her seizure threshold or even precipitated her seizures.

Toxicology consultation in seizures of unclear etiology can help discern drugs that offend even in therapeutic doses. Sudden onset of back pain during sleep can be an important clue to an unwitnessed seizure, which can cause vertebral compression fractures, even in the absence of a fall or other trauma. With the high volume of seizurerelated consults that toxicologists participate in, it is important to recognize this potentially serious complication.

\section{References}

1. Dunner DL et al (1998) A prospective safety surveillance study for bupropion sustained-release in the treatment of depression. J Clin Psychiatry 59(7):366-373

2. Johnston JA et al (1991) A 102-center prospective study of seizure in association with bupropion. J Clin Psychiatry 52 (11):450-456

3. Spiller HA et al (1997) Prospective multicenter evaluation of tramadol exposure. J Toxicol Clin Toxicol 35(4):361-364

4. Takahashi T et al (2002) Seizure-induced thoracic spine compression fracture: case report. Surg Neurol 58(3-4):214-216

5. Kalaci A et al (2008) Thoracic spine compression fracture during isoniazid-induced seizures: case report. Pediatr Emerg Care 24 (12):842-844 